\title{
Transport-based image reconstruction in turbid media with small source-detector separations
}

\author{
Andrew Dunn and David Boas \\ NMR Center, Massachusetts General Hospital, Harvard Medical School, Charlestown, Massachusetts 02129
}

Received June 28, 2000

\begin{abstract}
We demonstrate a new method for imaging through several millimeters of a turbid sample with a resolution of approximately $100 \mu \mathrm{m}$ by combining aspects of confocal reflectance microscopy and diffuse optical tomography. By laterally displacing the pinhole aperture of a confocal microscope we can achieve small source-detector separations and detect minimally scattered light. A reconstruction algorithm based on the first Born approximation to the radiative transport equation is then used to reconstruct an image of a $100-\mu \mathrm{m}$ absorbing object located $2 \mathrm{~mm}$ beneath the surface. (C) 2000 Optical Society of America

OCIS codes: $170.3010,170.1790,170.0180,170.3660$.
\end{abstract}

Noninvasive optical imaging in turbid samples such as biological tissues has been investigated extensively on both microscopic and diffuse spatial scales. Microscopic methods such as confocal reflectance ${ }^{1}$ and multiphoton fluorescence microscopy ${ }^{2}$ provide depth-resolved, micrometer-resolution images of tissue structure and function but are limited to depths of less than several hundred micrometers. Optical coherence tomography ${ }^{3}$ can penetrate to greater depths $(\sim 1 \mathrm{~mm})$ but at the expense of lower spatial resolution $(5-20 \mu \mathrm{m})$. The limitations on the penetration depths of these microscopic methods arise from the fact that each relies on the detection of singlescattered light, and since tissues are highly scattering, the probability of detecting single-scattered light through several hundred micrometers becomes prohibitively small.

Imaging with diffuse light, however, typically yields spatial resolution of the order of several millimeters to a centimeter, with penetration depths of several centimeters. Since the detected light has been scattered many times, an image-reconstruction algorithm must be employed to reconstruct scattering and absorption perturbations. Various reconstruction strategies have been used, ${ }^{4}$ and most are based on the diffusion approximation to the Boltzmann transport equation.

The spatial regime lying between that covered by the microscopic and the diffuse techniques remains largely unexplored, however. This is in part due to the complexity of the description of light interaction with tissue on a spatial scale of only a few scattering lengths. A few groups have attempted to examine this regime, ${ }^{5,6}$ but those studies have involved nonimaging approaches primarily intended for extraction of optical properties. A new imaging modality with the ability to penetrate depths greater than those attainable by microscopic methods with resolution significantly greater than the diffuse methods can be accomplished by combination of certain aspects of the microscopic and the diffuse methods. Such a technique could be widely used in a number of biomedical applications, such as functional imaging of the cerebral cortex through an intact skull in small animals and endoscopic imaging of small lesions too deep for conventional microscopic techniques to image and too small for diffuse methods to resolve.

Figure 1 illustrates the geometry used to image in the regime in which light is multiply scattered but not yet diffuse. By translation of the pinhole and detector that are typically used in confocal reflectance measurements to a position that is laterally displaced from the optical axis, the pinhole is imaged to a point that is laterally displaced from the source. Therefore the amount of lateral displacement of the detector aperture determines the effective source-detector separation. Because the source is no longer imaged onto the detector aperture, the detected light has been scattered two or more times.

As the source-detector separation increases, the detected light will have probed a deeper and larger region of the sample. The sensitivity of the measurement to a particular region of the sample can be maximized by variation of several parameters in the geometry of Fig. 1. These parameters include the source-detector separation (aperture offset), numerical aperture, depth of focus into the medium, wavelength, and pinhole diameter. In general, the size of the pinhole aperture will be larger than that used in confocal reflectance measurements, since it will be more important to maximize the signal intensity than to provide a large degree of spatial filtering.

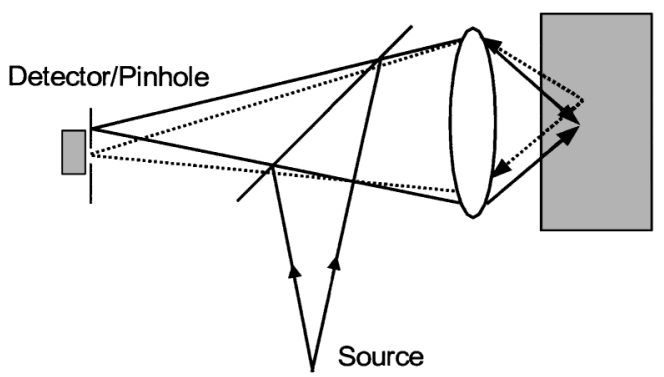

Fig. 1. Geometry for imaging at small source-detector separations. 
Direct imaging of single-scattered light by use of the geometry of Fig. 1 is not feasible. Therefore, to form an image, one must solve an inverse problem as in the diffuse regime. Since the diffusion approximation is no longer valid owing to the close proximity of the source and the detector, a description based on the transport equation ${ }^{7}$ must be employed.

To reconstruct an image of an absorption perturbation with a source-detector separation of only a few scattering lengths, we use a linear reconstruction algorithm based on the first Born approximation to the radiative transport equation. In the first Born approximation, the absorption and scattering coefficients are written as a sum of a homogeneous background component and a spatially varying perturbation, $\mu_{a}(\mathbf{r})=\mu_{a}{ }^{o}+\delta \mu_{a}(\mathbf{r})$ and $\mu_{s}(\mathbf{r})=\mu_{s}{ }^{o}+\delta \mu_{s}(\mathbf{r})$. The radiance, $L$, is then expressed as the sum of a background and a perturbed component, $L=L_{0}+L_{1}$. For an absorption perturbation the zeroth- and first-order terms, $L_{0}$ and $L_{1}$, are given by

$$
\begin{aligned}
L_{0}\left(\mathbf{r}_{d}, \hat{\Omega}_{d}\right)= & \iint S(\mathbf{r}, \hat{\Omega}) G\left(\mathbf{r}, \hat{\Omega} \mid \mathbf{r}_{d}, \hat{\Omega}_{d}\right) \mathrm{d} \hat{\Omega} \mathrm{d}^{3} r, \\
L_{1}\left(\mathbf{r}_{d}, \hat{\Omega}_{d}\right)= & -\iint \delta \mu_{a}(\mathbf{r}) L_{0}\left(\mathbf{r}_{s}, \mathbf{r}, \hat{\Omega}\right) \\
& \times G\left(\mathbf{r}, \hat{\Omega} \mid \mathbf{r}_{d}, \hat{\Omega}_{d}\right) \mathrm{d} \hat{\Omega} \mathrm{d}^{3} r
\end{aligned}
$$

where $G\left(\mathbf{r}, \hat{\Omega} \mid \mathbf{r}_{d}, \hat{\Omega}_{d}\right)$ is the Green function solution for the transport equation for a particular detector configuration. We note that perturbations in the scattering coefficient and the phase function can be computed with the same approach. Equation (2) illustrates that one of the primary differences between this approach and one based on the diffusion approximation lies in the angular dependence of the radiance. In this Letter we consider only reconstruction by an absorbing object by use of Eq. (2) and note that one can follow the same procedure to reconstruct a scattering perturbation.

To reconstruct an image of $\delta \mu_{a}(\mathbf{r})$ from Eq. (2), we compute $L_{0}$ and $G$ for a homogeneous background of known optical properties, using a Monte Carlo simulation in a focused beam geometry. ${ }^{8}$ We compute the Green function, $G\left(\mathbf{r}, \hat{\Omega} \mid \mathbf{r}_{d}, \hat{\Omega}_{d}\right)$, by simulating the propagation of light from the detector to the medium and then utilizing reciprocity to determine the fraction of light reaching the detector from direction $\hat{\Omega}$ at point $\mathbf{r}$ in the medium. Once the background radiance and the Green function have been computed for all source-detector pairs, an image of $\delta \mu_{a}(\mathbf{r})$ is reconstructed by use of the measured values, $L_{1}$, which are obtained for simulated data in this Letter.

Before Eq. (2) is used to reconstruct an image, the validity of the first Born approximation for the transport equation must be established. To test its validity, we compared the perturbed signal computed by use of Eq. (2) with the perturbed signal computed by use of a perturbative Monte Carlo simulation of an absorbing object embedded in a homogeneous background. ${ }^{9}$ The absorbing object used in the comparison was a cubic object with dimensions of $100 \mu \mathrm{m}$ located at a depth of $1 \mathrm{~mm}$ beneath the surface. The optical properties of the background and perturbation were $\mu_{s}=10 \mathrm{~mm}^{-1}$, $\mu_{a}=0.001 \mathrm{~mm}^{-1}, g=0.9$, and $\delta \mu_{a}=0.1 \mathrm{~mm}^{-1}$. The perturbed signals computed with Eq. (2) and with the full perturbative Monte Carlo model as the absorbing object was laterally translated through the sample are plotted in Fig. 2 for source-detector separations of $500 \mu \mathrm{m}$ and $2 \mathrm{~mm}$. The amplitudes of the perturbed signals in Fig. 2 have not been normalized, and they demonstrate that the absolute amplitudes of the perturbed signals are accurately predicted with the linear perturbation model. The comparison illustrates that the first Born approximation accurately predicts the perturbed signal and that Eq. (2) can be used to reconstruct an image of $\delta \mu_{a}(\mathbf{r})$. Since the first Born approximation is a linear approximation, the magnitude of the perturbed signal is directly proportional to the magnitude of $\delta \mu_{a}$. We have found that the linear approximation begins to deviate from the value predicted by the full perturbative Monte Carlo at $\delta \mu_{a} \sim 1.5 \mathrm{~mm}^{-1}$ for a $100-\mu \mathrm{m}$ object located at a depth of $1 \mathrm{~mm}$. In general, the value at which the linear approximation breaks down will vary depending on the size and depth of the perturbation. We have found that the maximum value of $\delta \mu_{a}$ increases as the depth of the object increases and decreases as the physical size of the perturbation increases, in agreement with standard perturbation theory.

Once the validity of the first Born approximation has been established (Fig. 2), we can construct an image of $\delta \mu_{a}(\mathbf{r})$ by writing Eq. (2) as a matrix equation of the form $y=A x$, where $y$ is the set of measurements and $x=\delta \mu_{a}(\mathbf{r})$. Truncated singular-value decomposition was used to invert this set of equations and find $\delta \mu_{a}(\mathbf{r})$ by use of simulated data. ${ }^{4}$

Reconstructed images of a $100-\mu \mathrm{m}$ absorbing object $\left(\delta \mu_{a}=1 \mathrm{~mm}^{-1}\right)$ at depths of 1 and $2 \mathrm{~mm}$ are shown in Fig. 3. The background optical properties of the medium were the same as those used in Fig. 2. The set of measurements used in the simulated reconstruction

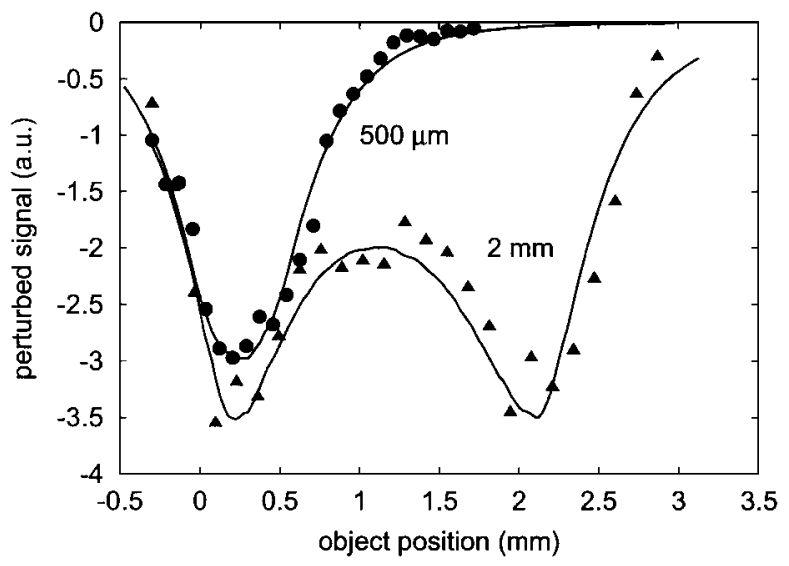

Fig. 2. Comparison of the perturbed signals computed with the first Born approximation (solid curves) and with a full perturbative Monte Carlo simulation (symbols) at source-detector separations of $500 \mu \mathrm{m}$ and $2 \mathrm{~mm}$ for an object located $1 \mathrm{~mm}$ beneath the surface with $\delta \mu_{a}=0.1 \mathrm{~mm}^{-1}$. 

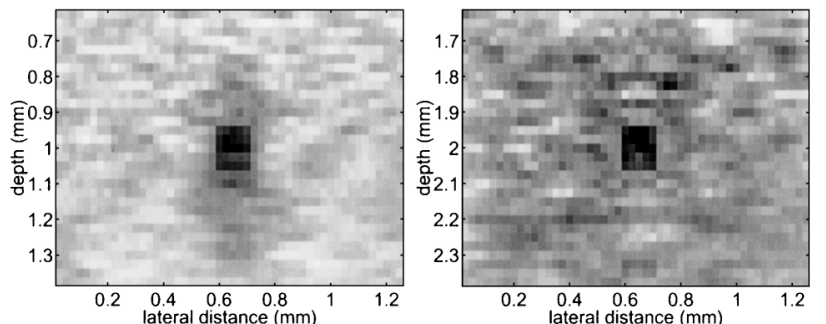

Fig. 3. Reconstructed images of a $100-\mu \mathrm{m}$ absorbing object located at depths of $1 \mathrm{~mm}$ (left) and $2 \mathrm{~mm}$ (right) below the surface, obtained from simulated data $\left(\delta \mu_{a}=0.1 \mathrm{~mm}^{-1}\right)$.

consisted of source-detector separations ranging from $400 \mu \mathrm{m}$ to $2 \mathrm{~mm}$ in $200-\mu \mathrm{m}$ increments at numerical apertures (NA's) of 0.2 and 0.4 , for a total of 18 source-detector pairs (9 with a source and detector $\mathrm{NA}$ of 0.2 and 9 with an NA of 0.4). Each pair was translated $1.25 \mathrm{~mm}$ across the surface of the sample in 51 steps of $25 \mu \mathrm{m}$, yielding a total of 918 measurements. The focal point of the source and detector was set to $1 \mathrm{~mm}$ beneath the surface for all measurements. The singular-value spectrum was truncated at 250 , and this number was determined by consideration of the $10^{3}$ signal-to-noise ratio for our simulation. The system was assumed to be shot noise limited, and the number of singular values was chosen so that the magnitude of the perturbed signal was greater than the measurement noise in the total detected signal (background + perturbation). The images in Fig. 3 clearly indicate that $100-\mu \mathrm{m}$ axial and lateral resolution is maintained to a depth of $2 \mathrm{~mm}$.

Based on the images of Fig. 3, it is clear that this method is capable of imaging in the spatial regime lying between the microscopic and the diffuse regimes. The ability to image through several millimeters of tissue with a resolution of a few hundred micrometers by use of this method should allow a new set of biomedical problems and applications to be addressed. For example, it should be possible to image small blood ves- sels located several millimeters within tissues, since the size of the vessels is a few hundred micrometers, and the difference in absorption between the blood vessels and the surrounding tissue will be $\sim 1 \mathrm{~mm}^{-1}$ in the near infrared. Another potential use of this method may be in optimizing the use of the indirect mode of the scanning laser ophthalmoscope ${ }^{10}$ for imaging subretinal structures, in which an annular aperture is used in place of a confocal aperture so that multiply scattered light is detected. Currently, the use of the indirect mode is based on observation rather than a theory or model based on light scattering in tissue. The model presented in this Letter could be applied to optimize the use of this mode.

The authors acknowledge support from the National Institutes of Health (grants NIH 1 P41 RR 14075 and NCI T32 CA09362). A Dunn's e-mail address is adunn@nmr.mgh.harvard.edu.

\section{References}

1. M. Rajadhyaksha, M. Grossman, D. Esterowitz, R. Webb, and R. Anderson, J. Invest. Dermatol. 104, 946 (1995).

2. W. Denk, J. Strickler, and W. Webb, Science 248, 78 (1990).

3. D. Huang, E. Swanson, C. Lin, J. Schuman, W. Stinson, W. Chang, M. Hee, T. Flotte, K. Gregory, C. Puliafito, and J. Fujimoto, Science 254, 1178 (1991).

4. S. Arridge, Inverse Probl. 15, R41 (1999).

5. F. Bevilacqua and C. Depeursinge, J. Opt. Soc. Am. A 16, 2935 (1999).

6. J. Mourant, J. Boyer, A. Hielscher, and I. Bigio, Opt. Lett. 21, 546 (1996).

7. A. Ishimaru, Wave Propagation and Scattering in Random Media (Academic, San Diego, Calif., 1978), Vol. 1.

8. A. Dunn, C. Smithpeter, R. Richards-Kortum, and A. J. Welch, Appl. Opt. 35, 3441 (1996).

9. A. Sassarolia, B. Blumetti, F. Martelli, L. Alianelli, D. Contini, A. Ismaelli, and G. Zaccanti, Appl. Opt. 37, 7392 (1998).

10. R. Webb, G. Hughes, and F. Delori, Appl. Opt. 26, 1492 (1987). 